\title{
How Are Programs Found? Speculating About Language Ergonomics With Curry-Howard
}

\author{
Johannes Emerich \\ johannes@emerich.de
}

\begin{abstract}
Functional languages with strong static type systems have beneficial properties to help ensure program correctness and reliability. Surprisingly, their practical significance in applications is low relative to other languages lacking in those dimensions. In this paper, the programs-as-proofs analogy is taken seriously to gain speculative insights by analysis of creation habits in the proof-centric discipline of mathematics. Viewed in light of this analogy, a sampling of mathematicians' attitudes towards formal proof suggests that the crucial role of intuition and experimentation in programming tasks may be under appreciated, hinting at a possible explanation of the challenges rigorously disciplined languages face in practical applications.
\end{abstract}

Categories and Subject Descriptors D.3 [Programming Languages]; K.2 [History of Computing]: Software

Keywords language ergonomics, type systems, programming systems, programs as proofs, propositions as types

\section{Introduction}

The thoughts in this paper grew over the course of a year, out of the opening remarks of my thesis defense in logic, as well as a series of informal discussions I have had since starting an industry job as a software developer. While I am in no way sure that what I am saying is authoritative or true, it has been my impression that my line of thought has been thought-provoking for those on the receiving end, and it is in this spirit that I decided to elaborate my thoughts into a form that might potentially reach a wider audience.

Being a student of logic with a professional and practical interest in computer programming, I was looking to write my thesis in an area that would gainfully combine both topics (Emerich 2015). The topic of type systems for programming languages was an obvious fit, but I soon faced with a puzzle: As a student of logic, everything about sound static type systems seemed obviously right, beautiful and true, and the research papers and languages I worked through made perfect sense. On the other hand, from my professional work in software development, I knew how little attention industry paid to the rightness, beauty and truth of this approach to programming. My puzzlement is certainly shared in large parts of the programming language theory community: If "types are the leaven of computer programming; they make it digestible" (Milner), they are light-weight formal methods that automatically prove "the absence of certain program behaviors" (Pierce) (both Pierce 2002), and we all want to write correct programs, why would anyone not use them? And especially, why would big, for-profit companies opt to not use them, or use such perversions of type systems as to render any guarantees worthless?

Industry practitioners seldom explain their motives but simply vote with their feet, or if they do explain, they explain in terms incomprehensible to PL researchers. It is not surprising then that the disregard for advanced type systems is often explained away by stupidity, carelessness, or lack of proper education. Without even wanting to dispute these labels, I started to wonder whom programming languages are created for. If a technological artifact is inadequate for its intended target audience and therefore its intended use case, it is unacceptable to blame the recipients of the artifact.

To illustrate, let us look at another discipline where technological artifacts are designed for a large audience, and which is often invoked in certain parts of the software engineering community: architecture.

In the wake of World War II, war-time destruction and the rapid growth of metropolitan areas necessitated the rapid provision of affordable housing. In countries all around the world, a solution was seen in modernist living machines. High-rise buildings for a new kind of communal living were built with great enthusiasm, and abandoned with great disappointment as many of the projects became attractors of poverty and living conditions rapidly deteriorated. New concepts for urban development with dense housing have incorporated feedback from this failing to create less fragile living environments for human beings. Another example of well- 
intended but ultimately failed architecture is Haus Wittgenstein in Vienna, a modernist building designed in part by philosopher Ludwig Wittgenstein. Few of his family members succeeded to live in this house for long, even while remarking on its beauty and elegance.

Would we find it convincing if the architects of modernist high-rise projects or Haus Wittgenstein blamed their dwellers for their inability to dwell in them? If they declared them too uneducated to live properly? I think not. Why then should we let programming language researchers off the hook, if their artifacts fail to meet the needs of the programming community?

What is to be done to learn about the needs of humans when constructing programs? Ultimately, this is an empirical question, requiring research and approximation by trial and error. To guide both processes, it is prudent to start with some judicious guessing. This speculative text is an attempt to judiciously guess plausible hypotheses from an analysis of $20^{\text {th }}$ century mathematics.

Section 2 introduces a formal connection between mathematics and computer programming, and questions the implied role of formal proof in mathematical practice. Section 3 stops at several points of historical significance in the past hundred or so years in mathematics, summarizing discussions about the interplay of intuition, formalism and rigor. Section 4 looks back at recurring themes found in said discussions, takes the programs-as-proofs analogy seriously, and defines some criteria to evaluate programming systems by. In section 5 these criteria are applied to a small number of recent proposals for programming languages and systems. Section 6 concludes.

\section{Programming in the Image of Mathematics}

Modern day research in type systems for programming languages occurs in fruitful collaboration with research in type theory and mathematical logic. Languages like Haskell and OCaml are part of the lineage of ML, a language grown out of a system for theorem proving. GHC, the Glasgow Haskell Compiler, supports a variety of extensions to Haskell's type system, that bring it closer to the powerful dependent type systems found in proof assistants like Coq and Agda. The connection between programming languages and mathematical logic is described succinctly in Per Martin-Löf's influential Constructive Mathematics and Computer Programming (Martin-Löf 1985):

Parallel to the development from low to high level programming languages, there has been a change in one's understanding of the programming activity itself. It used to be looked (down) upon as the rather messy job of instructing this or that physically existing machine, by cunning tricks, to perform computational tasks widely surpassing our own physical powers, something that might appeal to people with a lik- ing for crossword puzzles or chess problems. But it has grown into the discipline of designing programs for various computational tasks, programs that have to be written in a formally precise notation so as to admit of automatic execution. [... ] It has made programming an activity akin in rigour and beauty to that of proving mathematical theorems. (This analogy is actually exact in a sense which will become clear below.)

The way in which the analogy is "actually exact" is by means of a Curry-Howard-style correspondence between a fictitious programming language (borrowing from ALGOL, PASCAL and LISP) and Martin-Löf's theory of types. What this correspondence really shows exactly is that in an adequate language, the activity of writing programs is equivalent to the activity of writing a formal proof 1 .

It is of course the unfamiliarity of the computer scientist or logician with actual mathematical practice that allows them to conclude that this is, without qualification, a good thing. If, however, we look at the history of mathematics in the last hundred or so years, we see that even in mathematics the role of formal proof is contested or at least much more complicated than commonly assumed.

The monograph The Mathematical Experience stems from 1981, close to Martin-Löf's 1985 paper. Therein, Hersh and Davis describe "the most mathematician-like mathematician' 2 to display "the discrepancy between the actual work and activity" and even "his own perception of his work and activity" (Davis and Hersh 1981, p. 34ff.). I will quote selectively, though at some length, to illustrate:

He rests his faith on rigorous proof; he believes that the difference between a correct proof and an incorrect one is an unmistakable and decisive difference. [...] Yet he is able to give no coherent explanation of what is meant by rigor, or what is required to make a proof rigorous. In his own work, the line between complete and incomplete proof is always somewhat fuzzy, and often controversial.

[...]

To his fellow experts, he communicates [his] results in a casual shorthand. "If you apply a tangential mollifier to the left quasi-martingale, you can get an estimate better than quadratic, so the convergence in the Bergstein theorem turns out to be of the same order as the degree of approximation in the Steinberg theorem." This breezy style is not to be found in his published writings. There he piles up formalism on top of formalism. Three pages of definitions are followed by seven lemmas, and, finally, a theorem whose hypothe-

\footnotetext{
${ }^{1}$ For a modern introduction and brief history, see (Wadler 2015).

${ }^{2}$ As was customary at the time, this most mathematician-like mathematician is styled as "he".
} 
ses take half a page to state, while its proof reduces essentially to "Apply Lemmas 1-7 to definitions A-H."

When asked for a definition of proof, he gives one as "cleared up by the logician Tarski [...] and some others, maybe Russell or Peano", but also says that "of course no one ever really does it. It would take forever!" The less one knows about formal languages and formal logic, the better, as "[that] stuff is all abstract nonsense anyway".

Now, would our ideal mathematician be happy to do his work in Martin-Löf's formal theory of types? One should think not. Then why are we surprised if the average programmer does not seem to take to an equivalent programming system?

We will, in the following, undertake a brief historical tour of mathematics to find some clues as to why mathematical practice does not conform to the ideal of formal proof.

\section{Intuition and Formal Proof in Mathematics}

We begin our analysis, abruptly, early in the $20^{\text {th }}$ century, with the foundational crisis in mathematics in full swing. In response to misunderstandings and embarrassing failures in the face of an ever more significant mathematics, mathematicians, logicians and philosophers tried to establish foundations that would confirm and guarantee their understanding of mathematics as the house of certainty. To restore the endangered reputation of mathematics as a prototype of the most rigid science, the goal had to be not only to axiomatize all of mathematics, but also to show that in the chosen axiomatization contradictions are in general impossible (Hilbert 1917, p. 411). Even Russell's generous endeavor of axiomatizing all of logic was not sufficient according to Hilbert, as it was still necessary to establish that every mathematical problem has a solution (no ignorabimus), that every solution can be verified, and to find a measure for the simplicity of a proof. To put this plan into action, mathematical concepts and practices such as propositions and proofs had to be reified into mathematical objects that could be studied with mathematical methods. The famous rigor of mathematics was to be applied to mathematics itself. The successes and failures of Hilbert's program are famous, but its reception was mixed from the onset.

\subsection{Poincaré on Intuition and Logic}

Of interest for our analysis is the stance of Henri Poincaré, who was at once appreciative of the quest for certainty and skeptical of the emphasis on formalism. In a 1900 essay, Poincaré identifies "two entirely different kinds of minds" among great mathematicians, "one sort are above all preoccupied with logic", advancing "step by step, after the manner of a Vauban who pushes on his trenches against the place besieged, leaving nothing to chance", and the other are "guided by intuition and at the first stroke make quick but sometimes precarious conquests" (Poincaré 2005, p. 1012). Poincare believed it was the "very nature of their mind which makes them logicians or intuitionalists", and while "[the] first are incapable of 'seeing in space', the others are quickly tired of long calculations and become perplexed". But both are "equally necessary for the progress of science", both "have achieved great things that others could not have done".

Poincaré however was not blind to the progressive shift towards formality and rigor, even among the "intuitionalists", as "their readers have required of them greater concessions". The reason for this shift is the recognition that intuition "cannot give us rigour, nor even certainty". This is why formalism and logical analysis are necessary to further progress, a kind of cleaning up and clarifying that prevents illicit jumping to conclusions. But "intuition must retain its role as complement, [...] or as antidote to logic", not just for the student but also the creative scientist. To see the unity in a mathematical problem, we need "a faculty which makes us see the end from afar, and intuition is this faculty" (p. 1018). Poincaré uses the example of the concept of a continuous function, which from the image of a continuous mark of chalk gradually turned into a construction "irreproachable in the eyes of the logician". But even the logician relies on some sort of intuition, only, as in the case of Hermite, "the most abstract entities were for him like living beings", allowing them to "perceive at a glance the general plan of a logical edifice". "In rejecting the aid of the imagination, which, as we have seen, is not always infallible, they can advance without fear of deceiving themselves. Happy, therefore, are those who can do without this aid! We must admire them; but how rare they are!"

Poincaré, it seems, was not among them. His thesis adviser characterized him as follows: "It must be said, if one wants to give an accurate idea of how Poincaré worked, that many points of [his thesis] needed correction or explication. Poincaré was an intuitif" (cited after Mclarty 1997). Mclarty states that many of Poincaré's publications offered crucial new insights, laying the foundations to whole fields, all while employing hasty (or no) proofs, and getting substantial details wrong that were later to be worked out by others. Poincaré's question, "Who would venture to say whether he preferred that Weierstrass had never written or that there had never been a Riemann?", should be restated for the reader: "Who would wish that there had never been a Poincaré?"

\subsection{New Math: Real Mathematics Comes to Schools}

The foundational crisis in the past, and its shocking revelations absorbed or ignored, parts of the formalists' results and spirit found their way into research mathematics. Probably the prototypical example of $20^{\text {th }}$-century formalist mathematics is the highly influential work of Nicolas Bourbaki, a group of predominantly French mathematicians. According to a member, Bourbaki was set up almost in opposition to the intuitif Poincaré, against the older generation that "had learned mathematics in the old-fashioned way", "it was not the fashion to value Poincaré at all" (Senechal 1998). 
In the 1960s, this spirit of renewal found its way into primary education in mathematics. Today still known (and often ridiculed) under the moniker "new math", various educational reform programs in the United States and Europe put set theory, Boolean algebra and further abstract nonsense on schools' curricula. A cautionary tale of educational reform implemented in haste and abandoned without much analysis or care, new math disappeared as quickly as it had been put on the agenda, as teachers felt unable to teach and parents unable to understand the new materials. The history of the new math is complex and frequently misconstrued (Phillips 2015, p. 145), but the reactions of contemporary mathematicians to its introduction provide interesting insight into their attitudes. Especially in the United States, the reform efforts led to controversies among professional mathematicians and scientists, triggered not by the new emphasis on and interest in mathematics education, but by the image of mathematics thought to be underlying the reform proposals (Phillips 2014).

Physicist Richard Feynman in a commentary on new math stresses the importance of freedom, experimentation and intuition in learning and practicing mathematics (Feynman 1965). While this spirit "does not appear in [a mathematician's] final proofs, which are simply demonstrations or complete logical arguments which prove that a certain conclusion is correct", it is present in the way that he works in "in order to obtain a guess as to what it is he is going to prove before he proves it". To give authority to his claim, he quotes a pure mathematician, J.B. Roberts:

The scheme in mathematical thinking is to divine and demonstrate. There are no set patterns of procedure. We try this and that. We guess. We try to generalize the result in order to make the proof easier. We try special cases to see if any insight can be gained in this way. Finally - who knows how? - a proof is obtained.

Mathematician and educator Morris Kline spent considerable effort arguing against the new curriculum, publishing both an essay, Logic Versus Pedagogy (Kline 1970), and a book, Why Johnny Can't Add (Kline 1974). His protest against the reform was motivated by a strong belief in the importance of intuition and gradual development in mathematical training and practice. The arguments in both texts make much use of historical developments and anecdotes, illustrating the imperfections and intuitive leaps present in the work of accomplished mathematicians. Kline presents as an example the development of calculus from the basic concept of "instantaneous rate of change of a function" (Kline 1970 p. 267) to the modern formally precise expression. He documents the vague and faulty conceptualization evident in the writings of Newton and Leibniz, and Newton's defense of his work against "overprecise critics" posing a threat to the "fruits of invention". The same aspect is highlighted in
Cauchy's use of differentiability where he had only assumed continuity, making, on the whole, substantial progress.

Like Feynman, Kline sees mathematics as "primarily a creative activity, and this calls for imagination, geometric intuition, experimentation, judicious guessing, trial and error, the use of analogies of the vaguest sort, blundering and fumbling" (p. 271). To counter the response that intuition plays this important role only in those new to a subject matter, Kline recounts the anecdote of "the professor who was presenting a logical proof to his class, got stuck in the course of the proof, went over to the corner of the blackboard where he drew some pictures, erased the pictures, and then continued the proof" (p. 280).

In the end Kline concedes, quoting Weyl, that "logic is the hygiene which the mathematician practices to keep his ideas healthy and strong".

\subsection{The Threat of Speculative Mathematics}

Our third episode is set in 1993-1994, a discussion held in the Bulletin of the American Mathematical Society and triggered by a call for action by Arthur Jaffe and Frank Quinn (Jaffe and Quinn 1993). The authors worried about a perceived trend of an increase in speculative mathematics, due in part to a cultural clash between mathematics and physics. A nearly categorical statement opens the paper: "Modern mathematics is nearly characterized by the use of rigorous proofs". This is qualified to say that "information about mathematical structures is achieved in two stages", in the first stage "intuitive insights are developed, conjectures are made, and speculative outlines of justifications are suggested", in the second "the conjectures and speculations are corrected; they are made reliable by proving them". The two-stage process is compared to physics, divided in theoretical and experimental physics. For Jaffe and Quinn, theoretical physics is analogous to the speculative, intuitive stage in mathematical discovery, while experimental physics is analogous to verification and proof of speculation. Where in physics there has been productive division of labor along those lines, Jaffe and Quinn decry what they see as an onslaught of speculative mathematics (which they call theoretical mathematics), arguing that the culture of mathematics is unprepared. A list of cautionary tales of speculative mathematics gone wrong is followed by a problem characterization: Speculative work easily goes astray for lack of corrections from rigorous proof, it hinders further work by causing confusion about which parts are reliable, it gives glory to the theorizers while leaving ungrateful cleanup work for others, and finally confuses newcomers. They end with a short list of prescriptions to amend the situation, mostly calling for explicit labeling of speculative work, and thus shifting culture to reserve some glory for the task of rigorous validation.

This call was answered in a later issue of the bulletin (Atiyah et al. 1994), in good numbers by some of the speculative perpetrators Jaffe and Quinn had reprimanded. I can only recommend reading the responses, as they provide a 
fascinating insight into the varieties of approaches and selfimages among researchers in mathematics. The reader following this advice will notice that my quotations in the following are highly selective, which should not be reason for concern, considering that my claim is not that every mathematician looks at intuition and formal proof in the way suggested, but only that at least some amount of accomplished mathematicians do.

We find the first commentator, Michael Atiyah, "agreeing with much of the detail", but rebelling "against their general tone and attitude", presenting "a sanitized view of mathematics which condemns the subject to an arthritic old age" (p. 178). "But if mathematics is to rejuvenate itself and break exciting new ground it will have to allow for the exploration of new ideas and techniques which, in their creative phase, are likely to be as dubious as in some of the great eras of the past. Perhaps we now have high standards of proof to aim at but, in the early stages of new developments, we must be prepared to act in more buccaneering style". Providing an example, "Hodge's own proof was essentially faulty because his understanding of the necessary analysis was inadequate. Correct proofs were subsequently provided by better analysts, but this did not detract from Hodge's glory".

In a scathing comment, Armand Borel refers to the periodic waves of innovation and rigorization/systematization that have been a permanent feature of mathematics, accompanied constantly by fears that one might overpower the other. "Of course, [...] no part of mathematics can flourish in a lasting way without solid foundations and proofs".

Benoit Mandelbrot finds "most of it appalling". For him, Jaffe and Quinn's proposal is reminiscent of the shunning of great intuitive mathematicians such as Lévy and Poincaré by the mathematical establishment. He even goes so far as to ask why there had been so few great intuitive researchers of recent, and speaks of a "flow of young people" who were "acknowledged as brilliant and highly promising; but they could not stomach the Bourbaki credo" and left mathematics.

Saunders Mac Lane responds with an anecdote about a discussion between Atiyah and himself, "about how mathematical research is done".

For Mac Lane it meant getting and understanding the needed definitions, working with them to see what could be calculated and what might be true, to finally come up with new "structure" theorems. For Atiyah, it meant thinking hard about a somewhat vague and uncertain situation, trying to guess what might be found out, and only then finally reaching definitions and the definitive theorems and proofs. This story indicates that the ways of doing mathematics can vary sharply, as in this case between the fields of algebra and geometry, while at the end there was full agreement on the final goal: theorems with proofs. Thus differently ori- ented mathematicians have sharply different ways of thought, but also common standards as to the result.

If only the same could be said about differently oriented programmers! (Mac Lane goes on to say: "The sequence of the understanding of mathematics may be: intuition, trial, error, speculation, conjecture, proof. The mixture and the sequence of these events differ widely in different domains, but there is general agreement that the end product is rigorous proofwhich we know and can recognize, without the formal advice of the logicians.")

We see a wide spectrum of opinions on the role of rigorous proof in professional mathematics. On one end, Mac Lane voices a clear stance against ungrounded speculation and a demand for proof as the golden standard, but paired with a laissez-faire attitude towards the creative habits of individuals. On the other, Mandelbrot finds it sufficient to put forward his discoveries as conjectures, arguing against a unified narrow conception of what is acceptable mathematics. What we find in common among all cited here, however, is insistence on the value and necessity of a multitude of intuitive approaches to mathematical creation.

\subsection{Interactive Theorem Proving}

We shall finally look at some contemporary trends in mathematics. A current issue that has been in the making for much of the twentieth century but only recently has found increased attention is the use of computer-aided proof environments to develop and verify completely rigorous formal proofs. With this, we loop back to Martin-Löf's theory of types discussed in the very beginning.

Our source is the recently published Type Theory and Formal Proof (Geuvers and Nederpelt 2014). The book builds up a formal system $\lambda D$ somewhat similar to Martin-Löf type theory, extending the Calculus of Constructions with constructs first introduced in the Automath system of N.G. de Bruijn. After having built up the system to a substantial degree, the real work begins, by formalizing a real mathematical theorem (Bézout's lemma) and its proof in the system, illustrating that it is adequate for capturing serious mathematical content. It may be noted that this shows the adequacy for formalizing an existing and known proof.

Chapter 16, Further perspectives uses observations from the proof of Bézout's lemma along with general considerations to reflect on the system $\lambda D$ and type-theory-based proof assistants more generally. We collect here some quotes and discuss the implied context under which proof assistants may be used. "The type theory $\lambda D$ provides a system in which mathematical definitions, statements and proofs can be completely spelled out in a very structured way that is still close to ordinary mathematical practice. This enables and facilitates the formalization of mathematics and the checking of its correctness. [...] The high level of precision of type theory greatly improves the level of correctness of the formalized mathematics: incomplete proofs, or proofs using il- 
legal logical steps, are not accepted" (p. 379). The diagram on page 381 is relevant, because it uses as inputs informal proof $p$ and informal statement $A$, thus assumes that both have been formed at this point. If however we have not yet fully formed $p$ and $A$, the fact that the "precision of $\lambda D$ guides the proof development" (p. 380) may come back to bite us. It is no doubt a good thing that at some point a precise guide will point to flaws in the details of the development, but doing so early may lead the user astray trying to verify a nonsensical side-show statement that could easily be falsified by a quick series of trial and error. The author has made this experience first-hand working with the Coq proof assistant.

Their chapter closes with a prognosis: "Proof assistants have not yet developed into a standard tool for mathematicians, but we strongly believe they will in the future" (p. 387). In the world of mathematics, there is a culture delineating the phases of discovery and rigorization that suggests methods for the discovery process and may indeed enable publications to spell out results for proof checkers in ways similar to how results are elaborated into semi-formal proofs today. We have seen that there is by no means agreement on this, but at least mathematicians don't generally assume they have to start with the formal process.

In programming however, I am not aware of a general agreement that problems have first to be solved in pen and paper before their "formalization" as a program is to be started. What is more, programming tasks frequently are embedded in real-world interactions that require experiments instead of just pen-and-paper simulations.

\section{Learning by Analogy}

We can summarize as follows: The $20^{\text {th }}$ century has seen increased interest in the foundations of mathematics and a better understanding of the notion of proof. While rigorous proof can be seen as emblematic of modern mathematics, completely formal proof has been a polite fiction or idealization in research mathematics. A vocal community of mathematicians insists on the continued importance of intuition for the further development of mathematical content and objects to a prescriptive straitjacket that they fear would stand in the way of creative discovery.

Just how does this affect the practice of computer programming? Like mathematical argumentation, program construction has undergone a series of rigorizations to prevent the final product from going wrong. The parallel is so pronounced that there is an exact formal correspondence between various formal logics and programming languages, and there is an important sense in which program construction is equivalent to proof construction. More precisely, it is equivalent to the construction of completely formal proof.

We have seen that in the mathematical community the activity of formal derivation is not generally seen as adequate for the creation of new mathematical content. This begs the question: How do programmers working in rigorous languages find programs?

Mathematicians, in my layman's eyes, have highly idiosyncratic methods of discovery, and there is no reason for programmers to be any more constrained. Indeed, when exempt from corporate restrictions, programmers are free to employ whichever methods they find helpful in discovery: whiteboard sketches, pen and paper traces, contemplation of denotational semantics, Node.js prototypes, etc. And no doubt, in the era before time-sharing and personal computers, offline algorithm design was the norm, and one can still find industry veterans today who claim to spend weeks developing programs without compiling. Today's generation of programmers however grew up on REPLs and the editrefresh-run loop of browser development. The expectation is that the computer is an interactive device providing instantaneous feedback. The computer acts as a laboratory for the discovery of programs.

It would certainly be easy to dismiss this approach as dilettantism, the mark of the amateur, and it is certainly true that experienced practitioners develop higher level reasoning for familiar problems. But it should be recalled that in order to understand a problem, mathematicians employ "experimentation, judicious guessing, trial and error, the use of analogies of the vaguest sort, blundering and fumbling" (Kline) and run through special cases (Roberts) in their blackboard or pen and paper labs. With a powerful computing machine on their desks, why would programmers not want to make use of it for exploring the problem space by means of experiment?

Quickly observing the behavior of a few specific instances can give a feeling for the active forces in a process. Being able to push a value through my program and see the resulting crash can be illuminating with an immediacy not found in a lifeless compile-time error. For a quick sanity check I can run a partial implementation early, even though I have not announced all my assumptions (invariants) to the compiler, because I know which precise input I will use. If these activities are useful and we deprive the programmer of such means of discovery, she will opt for a system in which the balance between discovery and comprehensibility is more to her (present) advantage.

\subsection{A Bad Proposal: Prototype in Lisp, Implement in Haskell}

One might think that we already have all the ingredients: Permissive dynamic languages and safe statically typed languages. So it is fine to fumble and blunder in Python or Lisp, but the real work needs to happen in a disciplined language. This does not seem to lead very far, however. The current trend in software development is towards agile and away from waterfall methods. Programs are not discovered in one creative act to then be reconstructed cleanly and rigorously. Software is often long-lived, undergoing constant evolution. 
A possibly stable and rigorous core needs to be seamlessly integrated with a more provisional, in-flux surface area.

It is often an additional requirement to be able to interact with existing software libraries, such that a separate prototyping environment becomes unwieldy. When asked about the motivation to replace the Structure and Interpretation of Computer Programs course with a Python-based course in the MIT undergraduate curriculum, Gerald Sussman responded that the type of engineering required to write software had changed in the 1990s. Instead of the analysis and synthesis view taught in SICP, what was now needed was a more experimental, science-like approach in which "you grab this piece of library, and you poke at it [...] see what it does" (Sussman 2016). Sussman readily admitted that the previous curriculum was more coherent, but engineering had changed, and it was necessary to find a new way to do and teach it, even if they were still in search of the right approach. Not just first-in-class teaching curricula, but also first-in-class programming systems need to adapt to the real contextual usage requirements.

\subsection{Gradual Rigor}

If rigorous methods in practical software construction are to succeed, they can not be hermetically sealed off from environments that allow for creative discovery and preliminary approximations. An ideal programming system would allow for a wide spectrum of program construction, granting freedom for the act of discovery and means for the hygiene of rigorization as an approach crystallizes.

As in mathematics, our goal should be enough rigor to keep our programs healthy and strong, but enough flexibility to enable us to fumble and blunder when still figuring things out. And like in mathematics, we should be able to move fluently, competently, and with confidence between both ends of the spectrum.

Realism about the work of the practitioner In a 1979 paper DeMillo, Lipton and Perlis (DeMillo et al. 1979) challenged the software verification community to move from a standard of perfection to a standard of reliability, arguing that even in mathematics "absolute rigor" had, in fact, not been attained, and even less so in applied engineering disciplines. They pointed to the supposed infeasibility of full verification by social processes such as the judging of proof by an expert audience. Type systems can be seen as a partial response to this problem, where verification is done automatically by machines instead of a human community. The promise of dependent type systems is to far extend the reach of possible correctness guarantees embedded in proofcarrying code (Asperti et al. 2009).

This line of research seems to provide very apt responses to the problem of verification described by DeMillo et al., by replacing the manual social verification step by machine verification, based on a codification of the accepted intersubjective standard. But this does not absolve of the question of how the design of languages with an eye towards easy automatic verification affects the resulting languages and its users as individuals for the purpose of initial creation. In this context, it will not do to give some principled theoretical account of possibility, but details of language ergonomics will have to be considered. A notorious polemic declares that untyped languages are unityped, and thus a mere special case of statically typed languages (Harper 2011, elaborated in Harper 2012). This clarification is highly interesting, but in no way accounts for the actual usage affordances of static and dynamic languages, respectively. Harper makes great points about the potential power of dynamicity safely integrated into a statically disciplined language, while at the same time giving nothing but condescending explanations for the popularity of dynamic languages.

In mathematics, we saw a folklore belief (or pretense?) that rigorous formal proof is at the center of the mathematician's practice, but considerable evidence that this is not the full story, and even found vocal individuals insisting on the importance and legitimacy of non-formal methods. Maybe the work of mathematicians is in principle equivalent to formal proof, but in the reality of the details, it is not. Maybe also, in the reality of the details, programmers find it easier to discover solutions to their tasks in the degenerate unityped languages they choose over languages with strong advanced type systems.

\subsection{The Landscape Today}

In today's mainstream programming language landscape, programmers have few options other than making a choice between languages with a simple and comprehensible mathematical foundation but with a tightly controlled execution model that disallows or disincentivizes direct experimentation, and languages which will allow for fumbling and blundering but do not offer a clear path towards a rigorous formulation with strong static correctness guarantees.

On one end of the spectrum, Haskell and OCaml are popular statically typed functional languages. Their semantics are relatively simple and amenable to mathematical reasoning, and they possess powerful static type systems that are still growing in expressivity. These are the languages that most seem to correspond to the vision of programming conjured up by Martin-Löf.

On the other end of the spectrum, scripting languages like Ruby, Python or JavaScript are conceptually less predictable and do not lend themselves to mathematical reasoning. The lack of compile-time type checking makes it easy to experiment and write programs by incremental approximation, but also prevents compile-time guarantees about program correctness, and disadvantages the language with respect to tools support.

Languages like Java, C\# or C++ provide static type systems that give them compile-time checks, superior tooling, and often superior run-time performance. On the other hand, they are not constructed around principles that allow for 
straight-forward application of modes of mathematical reasoning. They possess type systems that still leave ample room for fumbling and blundering, but provide little help in eventually leaving that stage.

Especially in the web programming sphere, there have been recent attempts to retrofit dynamic languages such as PHP, JavaScript and Python with static type systems, which often leads to idiosyncratic design choices. Dart and TypeScript sacrifice soundness of the type system to use "optimistic" type compatibility rules, in the interest of making static types easier to use. Runtime errors are trapped by the underlying dynamic language (Brandt 2011). Similarly flawed type systems have appeared before, if with less pride, for example in Java.

If we want to take the programs as proofs metaphor seriously, not just as a theoretical construct, but as something that can actually serve as a practical model for programming, we need to think about ways of combining the best properties of the various systems, or come up with altogether new ways of supporting the modern development process.

\subsection{A Speculative Evaluation Framework}

For programming systems that support development all the way from initial creation to eventual rigorous codification, we expect supporting capabilities in two phases: discovery and codification.

\subsubsection{Discovery}

The responses to Jaffe and Quinn hinted at the high diversity of cognitive styles and approaches among mathematicians. While ultimately mathematicians have a rigorous common standard of communication, there is a lot of freedom for individuals to work with their preferred methods and amount of guesswork. The degrees of freedom a technological system can provide may always need to be augmented with activities external to the system, yet programming languages could optimize for the level of flexibility possible when already rigorously formulated parts of a software system interact with experimental parts. We define three criteria that are likely to be useful in exploring a problem space and discovering possible solutions by enabling fumbling and blundering.

Special cases Testing a procedure by applying it to only some special cases is irresponsible, but trying a basic idea first on regular, then on edge cases is a time-tested heuristic for developing the first draft of a general procedure. Considering special cases allows local and concrete reasoning in place of contemplation of abstract generalities. A programming environment can allow programmers to run and observe partial implementations on hand-selected inputs, even if the implementation has obvious holes that have yet to be addressed.

Flow trumps flaw An uninterrupted flow of ideas and associations is more important than the premature attention to flawed details. I need not work out the details of an approach I discard after five minutes of exploration.

Today's incarnations of rigorous languages are not very close to absolute rigor. In most programs, there are many properties that are not captured by the type system (list length, effects, co-effects), and I don't have to battle the type checker on those dimensions. Nevertheless, there is a variety of cases where the type checker insists on guarantees for certain properties. The more the type system conforms to the ideal of preventing programs from going wrong, the more details will have to be explicitly stated and treated.

While the details need to be taken care of as the overall picture clears up, it is important to develop a high-level understanding and see an idea through enough to evaluate its overall value, before investing the time to care about all the details.

Partial execution Especially when making changes to an existing codebase, the programmer may want to test the changes to one part of the program, while ignoring - consciously or unconsciously - that global invariants have been broken. The environment can allow the execution of partially functional programs, even if there is no global coherence.

\subsubsection{Codification}

Discovery and experimentation are important, but so is hardening and gradual refinement towards a shared and practically verifiable standard of correctness. Not only does this provide better means for creating reliable artifacts, but it also is the basis for a common language to communicate ideas and judge implementations.

Crystallization As understanding of the programming task and software system crystallizes, the programmer should have a clear standard of correctness to work towards, and should constantly be supported to make the assumptions and invariants explicit and subject to verification.

\section{Tools for Discovery}

The goals and criteria described in the preceding section set up desiderata but do not prescribe any particular solution. A basic question that is left open, is whether we need to think of this as strictly a programming language problem, or whether it can be framed more broadly as a programming system problem. The idol of mathematics presents a pattern where the final form of rigorous proof is fixed (under adequate idealization), but any path that produces this form is considered legal.

In program construction, the tools for experimentation, discovery and crystallization can either be built into the language, or be available in a programming environment that allows to eventually produce a program in a final rigorous form, even if the language itself has no concept of imprecision. Both approaches have been explored in prior work, and it makes sense to discuss examples viewed through the lens of this distinction. 


\subsection{As Programming Language Problem}

A programming language that is to support the development process from experimentation to refinement into a well-understood, rigid form, needs to possess a powerful type system that can capture the relevant behavioral properties, but also allows to encode and tolerate a level of indeterminacy to support the phases where the programmer is unwilling or yet unable to formally explain their intention in detail.

Without reference to a particular implementation strategy, this general idea of viewing the static-to-dynamic continuum as a language-internal problem has already been called for in (Meijer and Drayton|2004).

Gradual Typing Gradual typing is an attempt to combine the benefits of static and dynamic type checking, yielding control of which parts of the program are statically checked to the programmer. At the core, gradual type systems extend static type systems with a special dynamic type that is similar to a global supertype (Object in Java) that also acts as a global subtype. To prevent the type hierarchy from collapsing under the subtyping relation, however, a new nontransitive consistency relation is introduced that allows any type to be implicitly converted to and from dynamic. The dynamic type can be associated with an expression either explicitly or through omission of type information, in which case the expression can act as any type at compile time, with dynamic checks for each operation performed at run-time.

By supporting both approaches in the same language, gradual typing enables programmers to evolve programs from an implementation with only dynamically checked types to a more predictable codebase with compile-time correctness guarantees. As the program evolves, fragments without static type checks can enter and be annotated repeatedly, or even remain part of the code base indefinitely.

The use of special cases is facilitated by the generous type conversions possible in the system, but may require manual effort on the side of the programmer. The ability to use dynamically typed fragments is supportive of programmer flow. Partial execution is possible, but limited to the dynamically typed fragments of a program. The presence of a clearly defined and enforced type discipline provides a framework for crystallization but relies on the programmer's discipline to make use of it.

The term gradual typing was introduced in (Siek and Taha 2006). Recent research has been focused on improving performance and communication of type errors (Garcia et al. 2016, Takikawa et al. 2015). Established languages that sup-

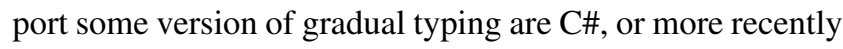
Dart and TypeScript.

Dependent Types The question of how much type information to extract from the programmer becomes more relevant as the expressiveness of type systems grows. In Why Dependent Types Matter, Altenkirch et al. present a series of refinements of merge-sort written in Epigram, a functional language with dependent types. In their words: "It is the programmer's choice to what degree he wants to exploit the expressiveness of such a powerful type discipline. While the price for formally certified software may be high, it is good to know that we can pay it in installments and that we are free to decide how far we want to go" (Altenkirch et al. 2005). Because of the expressive type system it is possible to encode not just structural information about function input and output, but more complex required and guaranteed properties for input and output - pre- and post-conditions. As an example, a vector concatenation function may carry the information that the length of the output vector is the sum of the input vectors. If the compiler is unable to infer this fact from the function definition, the programmer may have a proof obligation on her hands! In this case, as an alternative to providing a proof of this simple fact, Epigram allows to use a more imprecise type that will merely express that the output vector has some length.

The ability to choose the level of detail of typings grants programmers the power to move more or less quickly and revisit parts of the code deserving of greater precision over time. This helps prevent disruptions of flow, and allows for straight-forward crystallization. It is possible to observe execution instances for selected special cases as long as they are permitted under the current level of type precision. Execution of any part of the program is only possible if the whole program compiles.

\subsection{As Programming System Problem}

Statically-Aided Discovery Instead of manually running experiments on code with lacking type information, programmers can provide type information and receive automatic and direct feedback from the programming environment that may help revise and refine types and implementation.

(Dybjer et al. 2003) presents an extension to the proof assistant Agda, that combines static and run-time analysis by means of property-based testing to quickly identify deadends in formal proofs with high probability. In this manner, the system user can gain confidence in the validity of her current attempt, before potentially wasting time and energy trying to prove some ineffable, false property. This approach supports crystallization by reducing the time to feedback. Flow is somewhat disrupted by switching from implementation to verification, but examples for testing are even automatically generated. Partial execution is limited to the fragment under consideration.

A similar system is described in Dynamic Witnesses for Static Type Errors (Seidel et al. 2016), which aims to complement the abstract error messages provided by OCaml's type checker with problematic example inputs and an accompanying trace that illustrate the source of the error. Special cases, that is example inputs and executions, are provided only for negative cases that produce a type error. Be- 
cause the tool is external to the target language and compiler, execution of partially erroneous programs and testing with arbitrary examples is otherwise not possible. Flow is disrupted in just the same way as with a regular compiler, but the duration of the disruption may be decreased. There is no added support for gradual crystallization, even while there is a clear and enforced standard of correctness.

There is a tradition of type-directed tools for interactive proof construction in proof assistants like Agda, Coq and Isabelle. A graphical user interface can present the available hypotheses and open proof obligations and allow to select standard routines (tactics) to automatically proceed with parts of the proof construction (see also Asperti et al. 2009; Geuvers and Nederpelt 2014). The same idea can be applied to aided program construction as suggested in (Altenkirch et al. 2005): The environment lists the current programming goals based on the available type information and the programmer either implements them manually or by means of a meta-programming rule available as library code.

Instead of supporting experimental discovery, the programming environment provides support in reasoning at the static level, gradually working towards a pre-defined and checked type-level description of each part of the program. The language Idris, designed for general purpose programming, has a compiler with explicit support for this style of interactive type-directed development (Brady 2016).

Pluggable Type Systems Type disciplines can be seen as entirely external to the programming language, in which case there may be not one, but many static type systems designed for a language, allowing the programmer to pick the most appropriate one or ones. Such optional, pluggable type systems that have no effect on language semantics were argued for in (Bracha 2004). Because pluggable type systems are essentially analysis tools for dynamic languages, all discovery-phase benefits for flow, special cases and partial execution transfer unimpinged. On the other hand, type systems become more of a tool for individual developers and there is no longer any notion of crystallization towards a common standard of rigor.

Flexible Execution Models for Typed Languages This category of approaches is closest to gradual typing in that a designated type system or standard of rigor is assumed and combined with the desire to emulate the development experience of dynamic languages. Unlike in gradual typing, however, the type system is not enriched to allow the distinction between static and dynamic fragments of a program. Instead, the programming environment is altered to (optionally) defer certain type checks to run-time, allowing the execution even of provably type-incorrect programs. Execution may fail with a trapped error if the execution path leads to an error, or continue without failure as long as no problematic part of the program is reached.

Always-Available Static and Dynamic Feedback (Bayne et al. 2011) notes that "[most] statically-typed languages embody the philosophy that an ill-typed program is of zero value" and henceforth reject such programs. Instead, Bayne et al. see such programs as valuable for experimentation and want to provide feedback from both static analysis and dynamic execution at any time.

Progress types (Politz et al. 2012), instead of giving the programmer control over which parts of the program are disciplined by a static type system, grants control over which type errors are to be compile-time errors, such that irrelevant errors can be ignored until they occur at run-time. The authors seem to see this approach as compatible with the idea of pluggable types, however the presented system is focused on the existing run-time type system of the base language.

Liberating the Programmer with Prorogued Programming (Afshari et al. 2012) aims at creating a new programming paradigm, based around a more interactive program creation workflow. Their approach is based around three principles: allowing the programmer to defer implementation partially to focus on an ongoing concern; allowing the programmer to supply appropriate values during execution; and allowing the programmer to execute partial implementations at any time.

In all three cases, disruptions to flow are minimal, special cases can be hand-picked, and execution can happen at will. With the possible exception of progress types, crystallization towards a statically-checked type discipline is an explicit goal and can be pursued gradually. An early version of the basic idea is described in (Cartwright and Fagan 1991). Recent versions of the Glasgow Haskell Compiler support the deferral of type checks to run-time (Vytiniotis et al. 2015).

\section{Conclusion}

There is much work left to be done, both in working out the details of even these speculative arguments, and if they convince, in empirical investigation of their implications. When reading the writings of researchers, one finds references to the popularity of so-called dynamic languages, but this often appears as a curious, brute fact, something not available to or worthy of analysis. Even publications presenting technologies in support of the dynamic habits of programmers seem to confine themselves to appealing to only that brute fact, putting the blame on programmers instead of justifying the benefits independently. To give just one example: "The ethos of gradual typing takes for granted that programmers choose dynamic languages for creating software" (Takikawa et al. 2015). My hope is to convince the reader that there is something to learn from looking at programs as proofs, and then looking at how proofs really come about, without falling prey to idolizing mathematics.

Looking at the two examples drawn from architecture and urban planning we can make a distinction that may help address a possible objection. On one side, Wittgenstein's work raises doubts about whether his design was guided by 
the ambition to provide housing ${ }^{3}$. On the other side, it seems plausible (if not necessary) to believe that the architects of modernist housing projects were in fact motivated by the desire to provide housing. Unfortunately, this does not mean that the results are altogether different in practice. Similarly, my claim need not be that language designers are aiming for a formalist paradise devoid of practical motivation. But even if for the best intentions their design takes inspiration from mathematics in a too simplistic manner, it may miss important aspects and lead to similarly formalistic outcomes.

If we focus too much on making "programming an activity akin in rigour and beauty to that of proving mathematical theorems", while losing track of the "rather messy job of instructing this or that physically existing machine, by cunning tricks, to perform computational tasks", and not even paying attention to how mathematicians really do prove mathematical theorems, we run the risk of erecting, like Wittgenstein, a dwelling for the gods. The reality of the practitioner is not as clean-cut and elegant as its idealization, but makes use of a variety of mindsets, cognitive modes, and good old fudging. Coming up with an account of how the rigorous formal systems we have conceived of can integrate with less rigid components will be challenging, but worthwhile.

\section{Acknowledgments}

The seed of doubt was planted during my graduate studies at the ILLC and further shaped by my work at Prezi. Andras Slemmer's interest prompted me to produce an elaborated written version. Jan Martin Raasch, Ignas Vyšniauskas and Christoph Gietl helped by discussing the written version at various stages. The reviewers caught some of my sloppier thinking and suggested further material and distinctions.

\section{References}

M. Afshari, E.T. Barr, and Z. Su. Liberating the programmer with prorogued programming. In Proceedings of the ACM international symposium on New ideas, new paradigms, and reflections on programming and software (Onward! 2012), ACM, New York, USA, 2012.

T. Altenkirch, C. McBride, and J. McKinna. Why dependent types matter. 2005.http://www.cs.nott.ac.uk/ psztxa/publ/ ydtm.pdf

A. Asperti, H. Geuvers, and R. Natarajan. Social processes, program verification and all that. In Mathematical Structures in Computer Science, volume 19, number 5, October 2009.

M. Atiyah et al. Responses to: A. Jaffe and F. Quinn, “Theoretical mathematics": Toward a cultural synthesis of mathematics and theoretical physics. In Bulletin of the American Mathematical Society, volume 30, number 2, April 1994.

\footnotetext{
${ }^{3}$ It brings to mind his metaphorical invocation of architecture: "I am not interested in erecting a building but in having the foundations of possible buildings transparently before me".
}

M. Bayne, R. Cook, M.D. Ernst. Always-available static and dynamic feedback. In Proceedings of the 33rd International Conference on Software Engineering, ACM, 2011.

G. Bracha. Pluggable type systems. In OOPSLA Workshop on Revival of Dynamic Languages, 2004.

E. Brady. Type-driven development with Idris. Manning, 2016.

E. Brandt. Why Dart types are optional and unsound. 2011. https://www.dartlang.org/articles/ design-decisions/why-dart-types

R. Cartwright, and M. Fagan. Soft typing. In Proceedings of the ACM SIGPLAN 1991 conference on Programming language design and implementation, June 24-28, 1991, Toronto, Canada.

J. Davis, and R. Hersh. The mathematical experience. Birkhäuser, Boston, 1981.

R.A. DeMillo, R.J. Lipton, and A.J. Perlis. Social processes and proofs of theorems and programs. In Commun. ACM, volume 22, number 5, May 1979.

P. Dybjer et al. Combining Testing and Proving in Dependent Type Theory. In D. Basin, B. Wolff editors, Theorem Proving in Higher Order Logics: 16th International Conference, TPHOLs 2003, Rome, Italy, September 8-12, 2003. Proceedings. Springer, Berlin, 2003.

J. Emerich. Applying types as abstract interpretation to a language with dynamic dispatch. Master's thesis. University of Amsterdam, The Netherlands, 2015.

R.P. Feynman. New textbooks for the "new" mathematics. In Engineering and science, volume 28, number 6, March 1965.

R. Garcia, A.M. Clark, and E. Tanter. Abstracting gradual typing. In Proceedings of the 43rd Annual ACM SIGPLAN-SIGACT Symposium on Principles of Programming Languages (POPL 2016). ACM, New York.

H. Geuvers, and R. Nederpelt. Type theory and formal proof. Cambridge University Press, Cambridge, 2014.

R. Harper. Dynamic Languages are Static Languages. Blog post. March 19, 2011. https: //existentialtype.wordpress.com/2011/03/19/ dynamic-languages-are-static-languages/

R. Harper. Practical foundations for programming languages. Cambridge University Press, 2012.

D. Hilbert. Axiomatisches Denken. In Mathematische Annalen, volume 78, number 1, December 1917.

A. Jaffe, and F. Quinn. "Theoretical mathematics": Toward a cultural synthesis of mathematics and theoretical physics. In Bulletin of the American Mathematical Society, volume 29, number 1, July 1993.

M. Kline. Logic versus pedagogy. In The American Mathematical Monthly, volume 77, number 3, March 1970. Mathematical Association of America, 1970.

M. Kline. Why Johnny can't add: The failure of the new math. Vintage Books, 1974.

P. Martin-Löf. Constructive mathematics and computer programming. In C A.R. Hoare and J. C. Shepherdson editors, Proc. of a discussion meeting of the Royal Society of London on Mathematical logic and programming languages. Prentice-Hall, Inc., Upper Saddle River, NJ, USA, 1985. 
C. Mclarty. Poincaré: Mathematics \& logic \& intuition. In Philosophia Mathematica, volume 5, number 2. Oxford University Press, 1997.

E. Meijer, and P. Drayton. Static typing where possible, dynamic typing when needed. In OOPSLA Workshop on Revival of Dynamic Languages, 2004.

C.J. Phillips. In accordance with a "more majestic order": the new math and the nature of mathematics at midcentury. In Isis, volume 105, number 3, September 2014. University of Chicago Press, 2014.

C.J. Phillips. The new math: a political history. University of Chicago Press, 2015.

B. Pierce. Types and programming languages. MIT Press, 2002.

J.H. Poincaré. Intuition and logic in mathematics. In W.B. Ewald editor, From Kant to Hilbert, volume 2. Oxford University Press, 2005.

J.G. Politz, H. Quay-de la Vallee, S. Krishnamurthi. Progressive types. In Proceedings of the ACM international symposium on new ideas, new paradigms, and reflections on programming and software (Onward! 2012), ACM, New York, USA, 2012.
E.L. Seidel, R. Jhala, and W. Weimer. Dynamic witnesses for static type errors. Electronically published on June 24, 2016: http://arxiv.org/abs/1606.07557 (to appear in print).

M. Senechal. The continuing silence of Bourbaki: An interview with Pierre Cartier. In M. Senechal editor, The mathematical intelligencer, volume 20, number 1, 1998.

J. Siek, and W. Taha. Gradual typing for functional languages. In TR-2006-06: Scheme and functional programming workshop, University of Chicago, 2006.

G. Sussman. Video recording. Electronically published on January 11, 2016: https://vimeo.com/151465912\#t=59m36s.

A. Takikawa et al. Towards practical gradual typing. In John Tang Boyland editor, 29th European Conference on Object-Oriented Programming (ECOOP 2015), volume 37. Schloss Dagstuhl, 2015.

D. Vytiniotis, S.P. Jones, and J.P. Magalhães. Equality proofs and deferred type errors: a compiler pearl. In Proceedings of the 17th ACM SIGPLAN international conference on Functional programming (ICFP 12), ACM, New York, USA.

P. Wadler. Propositions as types. In Communications of the ACM, volume 58, number 12, December 2015. 